\title{
Locally Linear Neuro-Fuzzy Estimate of the Prosthetic Knee Angle and its Validation in a Robotic Simulator
}

\author{
Arash Arami, Member, IEEE, Natacha Vida Martins, and Kamiar Aminian, Senior Member, IEEE
}

\begin{abstract}
In this work we presented a low-power magnetic measurement system based on only two Hall-effect elements and a permanent magnet integrated into a smart knee prosthesis to accurately measure knee flexion-extension. The smart prosthesis was affixed in a robotic knee simulator that provides the squat movements, and also replicates different patterns of recorded gait from subjects. The squat movements were used to build linear and locally linear neuro-fuzzy estimators to translate the magnetic measurements into knee flexion angle. The simulated gait patterns then used to validate the models. The locally linear neuro-fuzzy estimator showed a clear benefit comparing to the linear regression models, by sequentially splitting the measurement space into subregions and find local fuzzy models for each region. The obtained RMS errors on test data were lower than $1.3^{\circ}$ for the neuro-fuzzy estimates representing smaller than $3 \%$ of range of rotation. The result was compared with the estimates from a previously-designed configuration of three 2-D anisotropic magnetoresistive (AMR) sensors which tested in mentioned setup. We showed that by using the neurofuzzy model for two Hall-effect elements, similar performance to the AMR-based estimator can be obtained while the power consumption can be reduced more than three folds.
\end{abstract}

Index Terms - Instrumented prosthesis, Knee flexionextension, Hall-effect sensor, Anisotropic Magnetoresistive sensor, neuro-fuzzy model

\section{INTRODUCTION}

$\mathrm{K}$ NEE joints are frequently affected by acute injuries and development of joint disease such as osteoarthritis (OA). $\mathrm{OA}$ is the primary cause of joint pain and disability particularly in the elderly population [1]. The risk of developing symptomatic knee OA during a person's lifetime has been estimated at $40 \%$ for men and $47 \%$ for women [2]. Since the populations of the developed countries are ageing and the rates of obesity are increased, a rise in rates of knee osteoarthritis is inevitable [3]. Knee arthroplasty is the most successful solution for knee OA where knee is replaced with

\footnotetext{
${ }^{\mathrm{T}}$ his paragraph of the first footnote will contain the date on which you submitted your paper for review. It will also contain support information, including sponsor and financial support acknowledgment. For example,

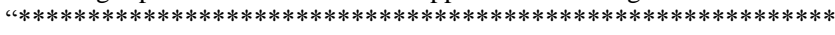
$* * * * * * * * *$,

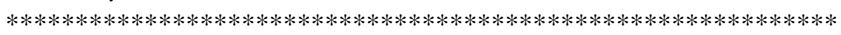

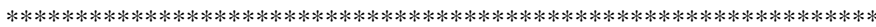

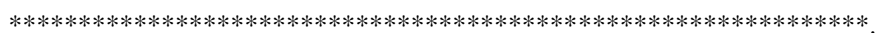

prosthetic components with the aim of relieving pain and restoring its function. Nowadays more than a million joints are replaced only in the EU and US [3], none provide information for the assessment of their functionality, and detection of deficits. This feedback should include the accurate estimation of kinematics and contact force distribution in the prostheses.

Recent studies in the area of instrumented prostheses are mostly focused on measurement of contact forces and moments [4]-[7], while the kinematics parameters left to be measured via external systems. However commonly used over-skin sensors or marker-based systems for estimating the joint angles and other kinematic parameters suffer from the soft tissue artifact (STA) [8]. STA adds subject-dependent and movement-dependent errors on measurements, and decreases their accuracy and precision. The RMS error of the Stereophotogrammetry motion capture system due to the STA based on measurements on several subjects were reported in stance phase of walking between 1.5 to 3.2 degree for knee Flexion Extension (FE) rotation [8]. This error during the activity of daily life was reported between 10 to $20 \%$ of range of FE [9], and the reported rigid STA error of FE angle during treadmill walking of 19 subjects was $1.6 \pm 0.5^{\circ}$ during the stance phase and $4.1 \pm 1.4^{\circ}$ during the swing phase [10]. The stereophotogrammetry measurement system restricts the measurement in laboratory environment, and cannot acquire the kinematics during the activity of daily life. Body-fixed inertial sensors (e.g. a combination of gyroscopes and accelerometers) were proposed for field measurements and tested on a number of subjects [11]. In addition to STA this technique also suffers from integration drift of angular velocity. Different approaches were proposed to obtain kinematics of the knee from inertial sensors [12], [13]. While these methods deal superbly with the drift of inertial sensors, they are still inaccurate due to the STA. Other skin-mounted sensors such as 3D magnetometers were proposed to measure the rotation based on the deviation from magnetic north [14]. Also a combination of 3D inertial sensors and 3D generatedmagnetic-field sensors was proposed to estimate the orientation of the body segments [15]. All these methods similarly suffer from the STA.

Intra-cortical pins were used in few studies to provide STAfree measurements, however this method is invasive. The alternative is to use X-ray fluoroscopy. Although this method provides STA-free measurement of the prosthetic joint 
kinematics, it suffers from constrained movement range, and $\mathrm{X}$-ray radiation which limits the number and duration of measurements. This is a laboratory-based method and cannot measure the kinematics during daily conditions.

While STA-free kinematics measurement during daily-life activities remains a challenge, recent progress in implanted sensors provides new opportunities, by taking advantage of the knee replacement to insert sensors into the implant for in vivo measurements of bone kinematics. Such smart knee prostheses were proposed in which the anisotropic magnetoresistive (AMR) sensors were embedded into the polyethylene insert [16], [17] to measure the magnetic field of capsulated permanent magnets in other components of the prosthesis. AMR sensors were previously chosen due to their higher resolution against Hall-effect sensors. The smart polyethylene also contained the force sensors, electronics and remote powering and wireless communication units [17]. No implantable battery was considered in the design since such existing batteries cannot meet the size and capacity requirements at the same time and not suitable for long term measurements. Instead, magnetic coupling remote powering was exclusively used for powering the sensors and electronics, while limited due to restriction on electromagnetic emission to living tissues [18]. There is therefore a remaining concern with regard to the power consumption of the sensors and electronics, and particularly the AMR sensors which solely consumed three times the obtained power budget [18].

To reduce power consumption a duty cycling of implanted sensors and their data fusion with complementary body-worn sensors was proposed and tested on numerically simulated data [19]. An alternative solution for the powering issue is to use lower-power sensors in expense of having lower resolution but use flexible models to improve the estimation of kinematics parameters of interest. However any solution to this issue needs to be validated in a more realistic manner than a numerical simulation. This study aims at providing an optimal solution in term of power consumption while maintaining high accuracy for STA-free measurements of knee kinematics by using implanted sensors. A minimal configuration of low-power Hall-effect sensitive elements is used together with estimation algorithms to provide a feasible alternative for the current designs. This sensor configuration along with two estimators were validated in a robotic knee simulator replicating human walking patterns.

\section{METHOD AND MATERIALS}

A mobile-bearing posterior-stabilized knee prosthesis (F.I.R.S.T, Symbios CH) was used in this study (Fig. 1) which consists of three main parts, a femoral component (FP), a tibial component (TP) and an ultra-high molecular polyethylene insert (PE). This prosthesis was instrumented by integrating the magnetic sensors and a permanent magnet, and fixed in our robotic knee simulator as described in the following subsection (B). The robotic knee generates actual knee rotations and validate the knee FE estimates against a reference motion capture system.

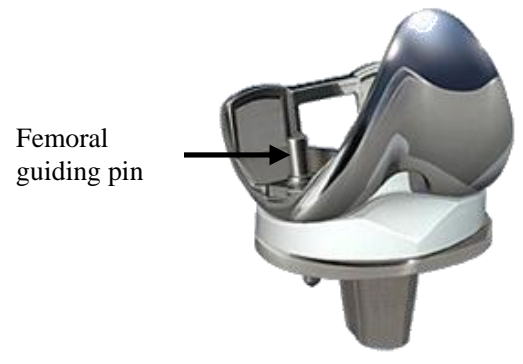

Fig. 1. F.I.R.S.T knee prosthesis, Symbios, Switzerland. This is a posterior stabilized mobile bearing prosthesis which includes three main parts. The bottom and top metallic parts called tibial and femoral parts respectively. Both mentioned parts have guiding pins to orient them on the tibia and femur bones respectively during the surgery. In the smart prosthesis a permanent magnet was capsulated into one of the femoral guiding pins. Middle polyethylene part (PE) housed the electronics including the sensors.

\section{A. Sensor Configuration}

Two 1-D Hall-effect sensors (HW-322B, Asahi, Japan) were used to obtain a low power consumption of $20 \mathrm{~mW}$, and configured in the PE. Also an NdFeB permanent magnet (S05-08-N, Supermagnet, Switzerland) was inserted in one of guiding pins of FP. The sensor configuration was designed based on a sensitivity analysis similar to the previous work [17]. In that analysis, the sensitivity of sensors placement in different points of $\mathrm{PE}$ to the $\mathrm{FE}$ rotation between $0^{\circ}$ to $80^{\circ}$ was estimated in a manually-operated knee simulator. The first sensor (S1) was located approximately where at zero flexion angle the magnet points at and the second (S2) near the anterior part of the polyethylene insert. The configuration then fixed in PE (Fig. 2) and then placed in a robotic knee simulator to simulate actual patterns of walking.

\section{B. Robotic Knee Simulator}

The robotic knee simulator was designed to validate the measurement of smart knee prosthesis in realistic conditions prior to the implantation in human subjects. This simulator includes a number of actuators to simulate the movement of hip joint or the application of body weight and to replicate the hamstrings and quadriceps muscle activities. Fig. 3 shows our mechanical designs and the realized knee simulator. Using this simulator, we can simulate different activity patterns with replicated realistic force and kinematics, however in this paper we focused on the FE during walking. We used two different gait patterns based on fluoroscopic measurements of a subject walking on treadmill [10] bearing the F.I.R.S.T prosthesis, and motion capture measurements of a subject walking over ground [20]. More details of these data are described in the validation subsection (D). These measurements were

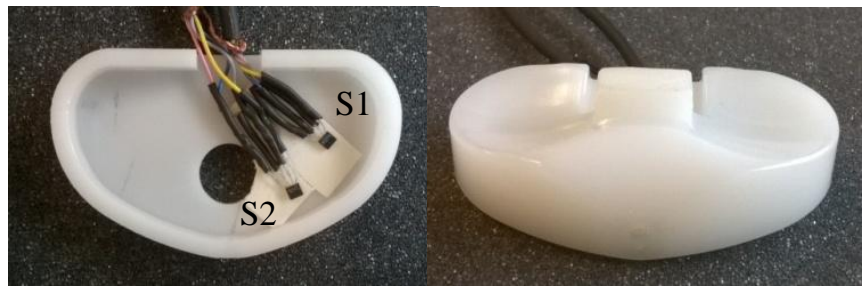

Fig. 2. Hall-effect sensor configuration in the polyethylene insert. 
preprocessed and used to estimate the knee angles. The knee angles were then approximated with Fourier series and fed into the robotic knee simulator control software, and replicated for the knee in sagittal plane using a hand-tuned closed loop Proportional Integral Derivative (PID) controller (Fig. 4). The femoral part with embedded permanent magnet, and the polyethylene insert with embedded Hall-effect sensors were fixed in the simulator (Fig. 3). The Hall-effect sensors were synchronized with the motion capture system that records the reference kinematics of the knee segments while simulating different gait patterns. The motion capture system consisted of four Mx3+ cameras (Vicon, UK) that are configured around the knee simulator to track the position of a set of markers attached to the simulator segments. This motion capture system provides the reference kinematic information for the validation purpose.

\section{Flexion-extension Angle Estimators}

Observing the nonlinear relationship between the raw signals of the sensors and the FE angle, two families of estimators were used, namely linear regression model with linear and nonlinear regressors, and locally linear neuro-fuzzy model built upon the raw data.

\section{1) Linear Regression Estimator}

A set of combinations and nonlinear functions of the raw measurements of Hall-effect sensors formed the candidate input pool. This set of possible regressors included the raw measurements, their one by one multiplication, and tangent hyperbolic of the raw measurements. Beside popularity of tangent hyperbolic as a bounded sigmoid-like function in feature extraction [21], this function was chosen to compensate the inverse-tangent-hyperbolic-like trend of magnetic data observed during the sensitivity analysis which was originally carried out for sensor configuration design. A
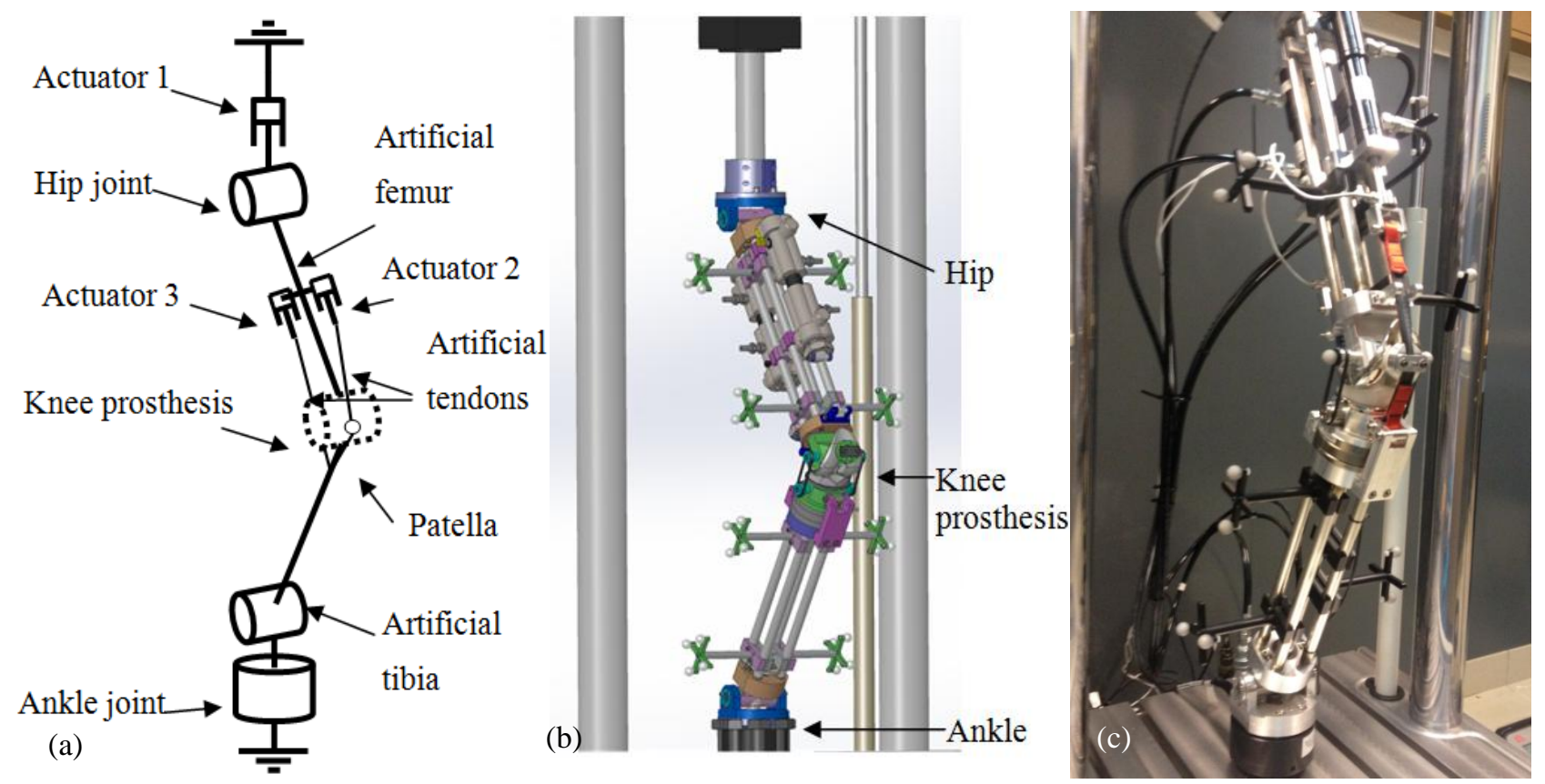

Fig. 3. EPFL robotic knee simulator: (a) abstract design, (b) mechanical design (SolidWorks, Fr), (c) mechanical implementation.
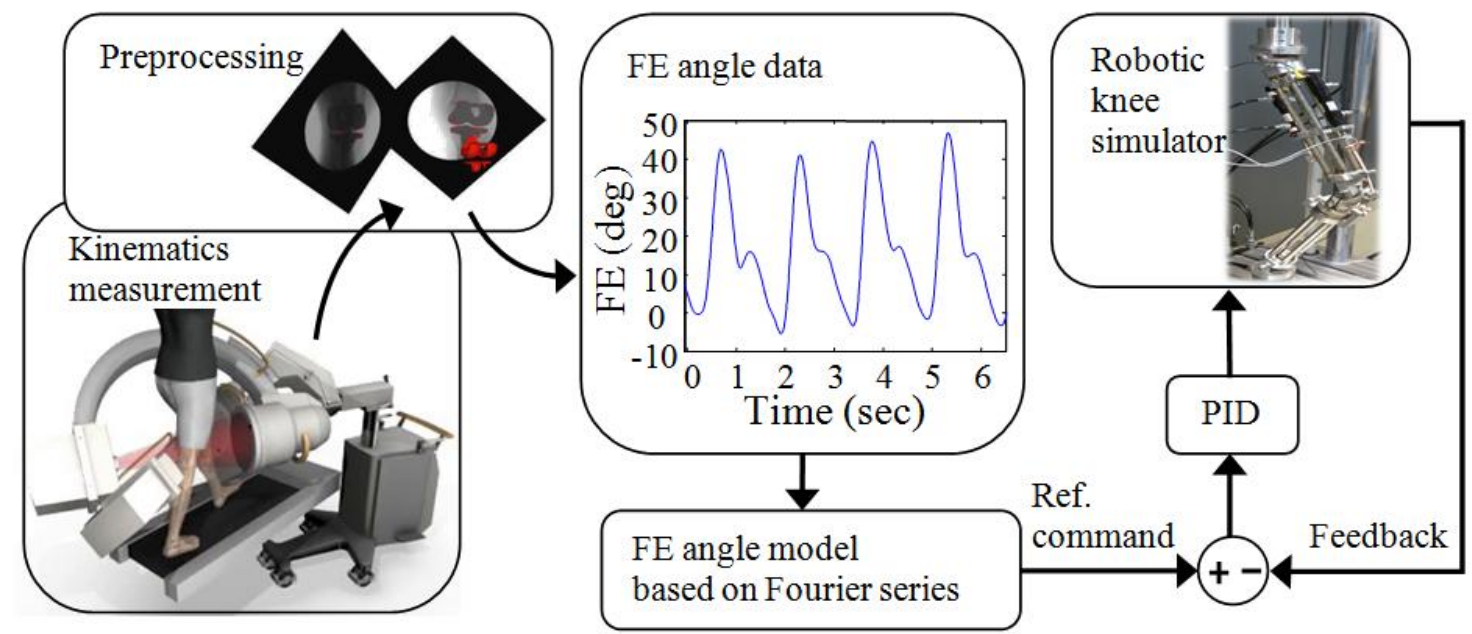

Fig. 4. Control schematic of robotic knee simulator based on realistic walking data. 
forward selection algorithm [21] was used on the train set to select the estimator's inputs from the pool. The highly correlated inputs with the FE angle, which have low correlation with others inputs were selected and then fed into a linear regression model. The model weights $(W)$ were estimated by ordinary least square on the train data (1).

$$
W=\left(X^{T} X\right)^{-1} X^{T} Y
$$

where $\mathrm{X}$ is the matrix of regressors and $\mathrm{Y}$ is the target angle in the train dataset. Linear regression model was chosen due to its simplicity and to be comparable with previous studies [16].

\section{2) Locally Linear Neuro-Fuzzy Estimator}

Locally linear models (LLM) split the measurement space into $M$ subregions where in each a linear model of measurements can be an accurate estimate of the target values. Considering the fact that different data points can be associated to any formed subregion with a degree of membership, a Takagi-Sugeno neuro-fuzzy model was used in this study to translate the measurements to the target values [21]. The membership of a data point to a region is close to one if the point falls close to the center of region and close to zero for the data points fall in the boarder of or far from the region. The neuro-fuzzy model has a merit over crisp locally linear models due to the sharing of different local models for each data point and capability of generating smooth mapping between input and output. This locally-linear estimator is an incremental model, which divides its $P$-dimensional measurement space into $M$ sub-regions, builds a validity function $(\Phi)$ and an LLM (represented by a set of weights $w_{i j}$ ) for each subregion. Then it employs all the LLMs and validities to generate the output estimate $(\hat{y})$ as calculated in (2) and depicted in Fig. 5.

$$
\hat{y}=\sum_{i=1}^{M} \underbrace{\left(w_{i 0}+\sum_{j=1}^{P} w_{i j} u_{j}\right)}_{y_{i}=L L M_{i}(\underline{u})} \cdot \phi_{i}(\underline{u})
$$

To exploit the fuzzy membership over the sub-regions, the neuro-fuzzy model associates a validity function for each LLM to each data point $\left(\underline{u}=\left[\begin{array}{llll}u_{1} & u_{2} & \ldots & u_{P}\end{array}\right]^{T}\right)$. The validity values for each point represent its fuzzy memberships to the existing LLMs. In this work we used Gaussian validity function centered at the center of each region $\left(\underline{c}_{j}=\left[c_{1 j} c_{2 j} \ldots c_{P_{j}}\right]\right)$ and with a diagonal covariance matrix. The validity functions are normalized such that sum of validities for each data point equals one (3).

$\phi_{i} \underline{(u)}=\frac{\mu_{i}(\underline{u})}{\sum_{j=1}^{M} \mu_{j}(\underline{u})}$

$\mu_{i}\left(\underline{u)}=\prod_{j=1}^{P} \exp \left(-\frac{1}{2}\left(\frac{\left(u_{j}-c_{i j}\right)^{2}}{\sigma_{i j}{ }^{2}}\right)\right)\right.$

where $\mu_{i}$ is a P-dimensional Gaussian function. Each non-zero element of the covariance matrix replicates the variance (spread of region) in a particular axis direction of the measurement space which was calculated as follows:

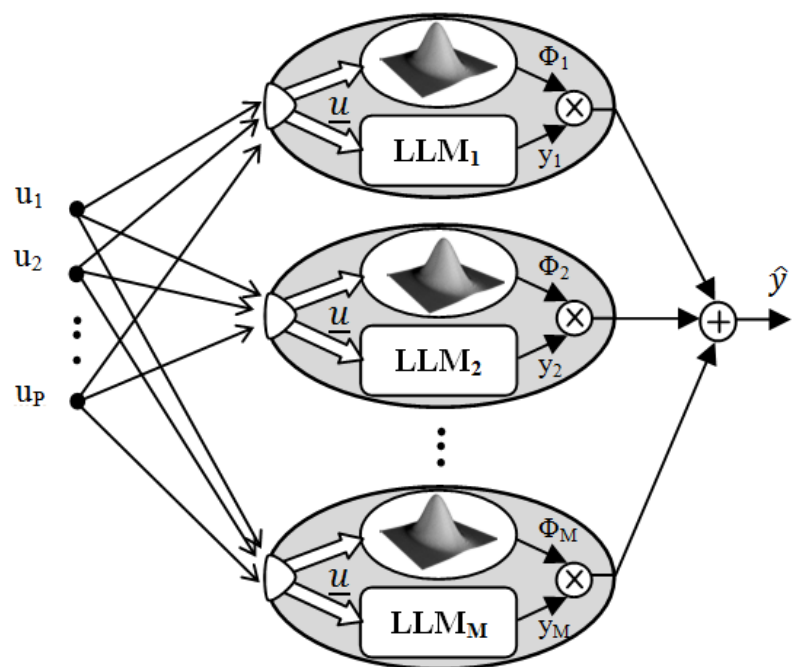

Fig. 5. Locally linear neuro-fuzzy model. $\mathrm{u}_{\mathrm{i}}$ is the $i^{\text {th }}$ dimension of each input data point. Each ellipse, a neuron of the model, contains a validity function and a locally linear model $\left(\operatorname{LLM}_{\mathrm{i}}\right)$. The data vector goes through the Gaussian functions and normalized to estimate the validity of data point for each LLM. These validity functions weight the output of LLMs. The weighted sum is the output estimate $(\hat{y})$.

$$
\sigma_{\mathrm{ij}}^{2}=\left(\frac{1}{3} \cdot \Delta_{i j}\right)^{2}
$$

where $\boldsymbol{\Delta}_{\mathrm{ij}}$ denote the length of the edge in the $\mathrm{u}_{\mathrm{j}}$ dimension (direction) of the $i^{\text {th }}$ region. LoLiMoT algorithm [21], [22] was used to split the measurement space to subregions, in which in each iteration the subregion with highest sum of square errors was chosen for splitting.

$$
\begin{aligned}
& I_{i}=\sum_{n=1}^{N} e^{2}(n) \cdot \phi_{i}(\underline{u}(n)) \\
& i^{*}=\arg \max _{i} I_{i}
\end{aligned}
$$

where $I_{i}$ denotes the local loss function of region $i$ computed by associating to the sum of squared error of the model, calculated on $N$ data points, to the region $i$ using its validity function. The results of possible axis-orthogonal splits, for the subregion with highest sum of square errors, considered as candidate new subregions as shown in Fig. 6. The LLMs for new candidate sub-regions were trained and used in the estimation of target in train data and the candidate which leads to the lowest sum of square error was taken as the split to be performed. In the present work, the raw measurements of the two sensors were used as the inputs and therefore the LoLiMoT algorithm was applied on a 2D measurement space.

\section{Validation}

The polyethylene insert with configured Hall-effect sensors was fixed into the robotic knee simulator that described in IIB. A squat movement, with an FE rotation from $14^{\circ}$ to $61^{\circ}$ was generated with the knee simulator while both PEintegrated Hall-effect sensors and the motion capture system were synchronously measuring the kinematics. The squat data were used as the training data to build the linear regression and locally linear neuro-fuzzy estimators to translate the 


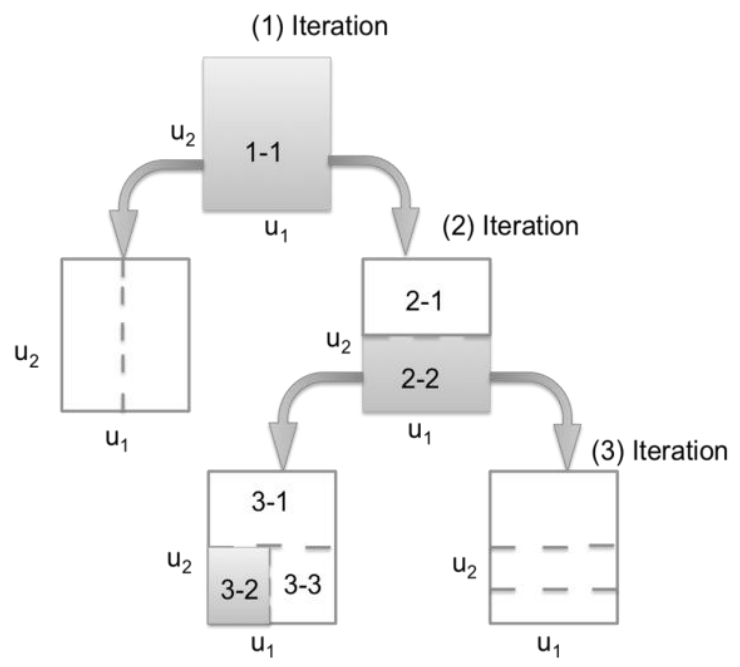

Fig. 6. Locally linear model tree (splitting procedure) in two-dimensional measurement space: at each iteration the sub-region with highest local loss function is chosen (gray), then possible axis-orthogonal splits will be applied, LLMs will be trained, and finally the splitting which leads to the lowest sum of square error will be kept. Subregion $\mathrm{i}-\mathrm{j}$ is the jth subregion at ith iteration.

magnetic measurements into $\mathrm{FE}$ angles obtained from the motion capture system. For testing the simulator, the gait patterns of two subjects walking over ground (Gait 1) and treadmill (Gait 2) from separate studies were extracted from the motion capture [20] and fluoroscopic measurements [10] with approximate flexion angle ranges of $46^{\circ}$ and $57^{\circ}$ respectively. Gait 1 data consisted of one overground gait cycle, was extracted from walking data collected from a subject during eight-month postoperative measurements with an instrumented prosthesis [20]. This gait pattern was repeated nine times with simulated random 5-degree amplitude variations. Gait 2 consisted of nine gait cycles on treadmill that were collected from a subject with F.I.R.S.T commercially-available prosthesis, 6 months after the surgery. Data were adapted and implemented in the knee simulator interface to replicate the knee movement for the two different gait patterns. The FE angle estimates obtained from the motion capture system (Vicon, UK) were used to evaluate the performance of the Hall-effect based estimators. The performance indices of mean (E), standard deviation (STD), and root mean square of error (RMS) and coefficient of determination $\left(\mathrm{R}^{2}\right)$ were used for comparison.

In order to compare the new Hall-effect based estimates with the previous AMR based estimates [16], an instrumented PE with three 2-D AMR sensors (HMC1512, Honeywell, US) was also fixed in the knee simulator and actuated with the same movement patterns. Similarly, a linear regression model with forward selection of inputs was built and used to estimate the $\mathrm{FE}$ angle in that case. Besides mentioned performance metrics, the signal to noise ratio (SNR) was computed for the both Hall-effect and AMR sensors.

\section{RESULTS}

The obtained SNR for Hall-effect and AMR sensors were $34.62 \mathrm{~dB}$ and $55.78 \mathrm{~dB}$ respectively. The selected inputs for the linear regression model via forward selection were as follows:

$$
X_{\text {selected }}=\left[\tanh \left(\mathrm{S}_{1}\right) \tanh \left(\mathrm{S}_{2}\right) \mathrm{S}_{1} \cdot \mathrm{S}_{2}\right]^{\mathrm{T}}
$$

A typical estimate of FE angle via linear regression estimator is depicted in Fig.7. The performances of this estimator on the train (Squat movement) and test data (different gait patterns) are shown in Table I. While the estimates were unbiased for the case of train data and Gait 2 in the test data, they had little bias (below $0.3^{\circ}$ ) in case of Gait 1 . The standard deviation of error in all cases were below $1.8^{\circ}$ with very high $\mathrm{R}^{2}$ (equal or greater than 0.99 ).

The performance of locally linear neuro-fuzzy estimator directly depends on the number of split by LoLiMoT algorithm on the measurement space, where the error on train data decreases by performing more splits. This is not however the case in test data, and too many splits would result in over fitting. Therefore, the algorithm stopped splitting as soon as the error on train data stopped decreasing drastically, which was after the seventh split. Fig. 8 shows the validity functions for the split space.

The performances of this estimator on the train and test data are shown in Table II. Also a typical estimate of FE angle is shown in Fig 7. The estimates on train data is unbiased with standard deviation error, which is equal to the RMS error, below $0.5^{\circ}$. The estimates of the test data are slightly biased (below $0.4^{\circ}$ ); however the standard deviation of error was always smaller than $1.3^{\circ}$. Similar to the linear regression estimators, the obtained $R^{2}$ are very high. The performance of locally linear neuro-fuzzy estimators was noticeably better than the linear regression both on the train and test datasets.

Fixing the PE insert with three embedded AMR sensors, in the knee simulator and performing the same patterns of gait in full speed, we compared the results with performance of previously proposed system [16]. This comparison is depicted in Table III for two different patterns of gait. An averaged

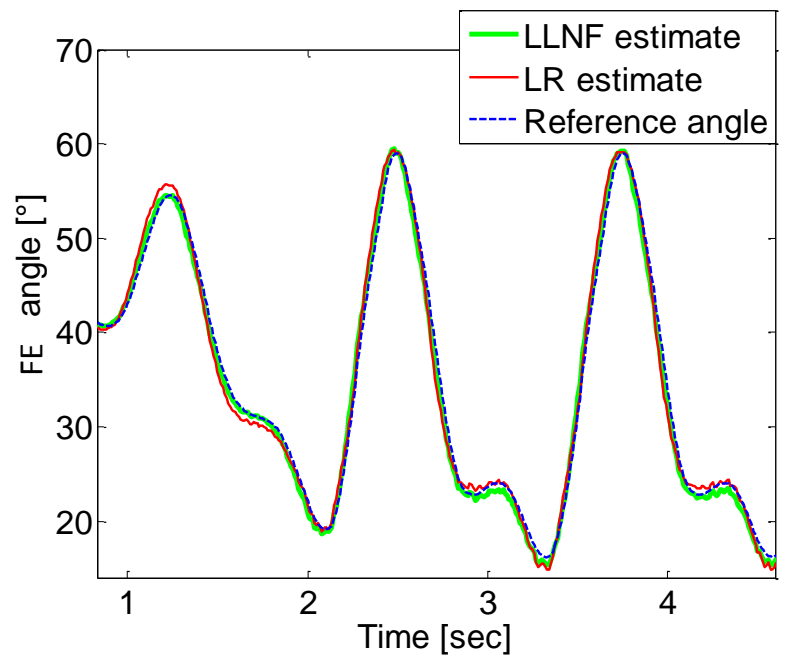

Fig. 7. A cut of a typical gait pattern simulated in the robotic knee simulator (blue) and estimated via the LR estimator (red), and LLNF estimator (green). Treadmill gait (Gait 2) during normal speed, i.e. self-selected speed by the subject, measured by biplane fluoroscopes. 


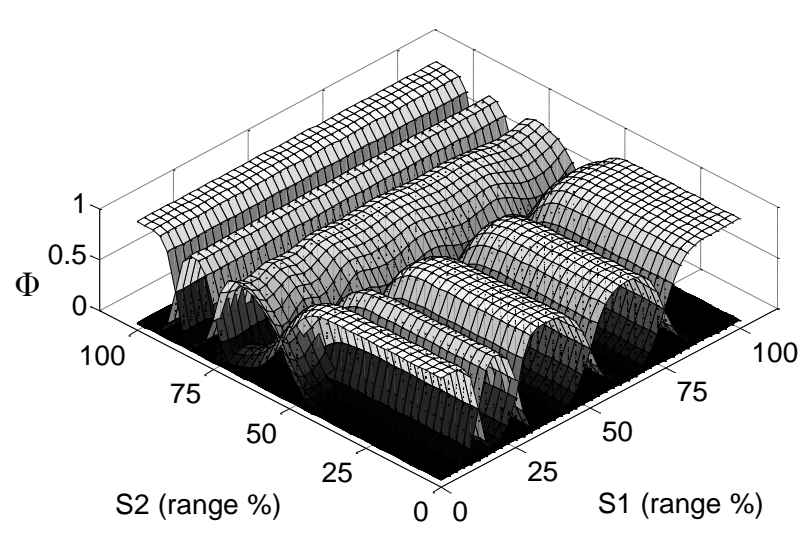

Fig. 8. Validity functions over the measurement space (product space of S1 and S2).The values of these function for each data point fall in measurement space show to what extent it is associated with each region and the corresponding LLM which is directly influence the estimation.

comparison over the data of both subjects, different gait patterns, is provided in Fig. 9. In this figure, E indicates the expected value of each performance metric over both gait patterns. This figure shows that LLNF estimator outperformed the linear regression estimators when using the Hall-effect sensors. The LLNF estimator however performed as well as previously proposed AMR-based system.

\section{CONCLUSION AND DISCUSSION}

In this study a configuration of two Hall-effect sensors embedded into the PE was used for STA-free estimation of the knee FE angle by help of different data-driven estimators. The instrumented PE was fixed in a knee simulator which generates realistic gait patterns and validated against a motion capture system. Two estimators, namely linear regression and locally linear neuro-fuzzy, were trained and tested in the knee simulator. They obtained great performances for FE angles
TABLE III. COMPARISONS OF PERFORMANCES ON TEST DATA BETWEEN THE NEURO-FUZZY ESTIMATOR OF FE ANGLES BASED HALL-EFFECT SENSORS AND THE LINEAR REGRESSION ESTIMATOR OF FE ANGLES BASED AMR SENSORS.

\begin{tabular}{lcccc}
\hline \hline & $\begin{array}{c}\text { Hall-effect sensor } \\
\text { based estimation } \\
\text { (LLNF) }\end{array}$ & \multicolumn{2}{c}{$\begin{array}{c}\text { AMR sensor based } \\
\text { estimation (LR) }\end{array}$} \\
\hline FE angles & $\begin{array}{c}\text { Gait1 } \\
\text { full }\end{array}$ & $\begin{array}{c}\text { Gait2 } \\
\text { full } \\
\text { speed }\end{array}$ & $\begin{array}{c}\text { Gait1 } \\
\text { sull }\end{array}$ & $\begin{array}{c}\text { Gait2 } \\
\text { full speed }\end{array}$ \\
& $0.32^{\circ}$ & $0.31^{\circ}$ & $0.35^{\circ}$ & $0.55^{\circ}$ \\
\hline E(error) & $1.25^{\circ}$ & $1.12^{\circ}$ & $1.17^{\circ}$ & $1.04^{\circ}$ \\
\hline STD(error) & $1.29^{\circ}$ & $1.16^{\circ}$ & $1.22^{\circ}$ & $1.18^{\circ}$ \\
\hline $\mathrm{RMS}$ (error) & 0.99 & 0.99 & 0.99 & 0.99 \\
\hline $\mathrm{R}^{2}$ & & & & \\
\hline \hline
\end{tabular}

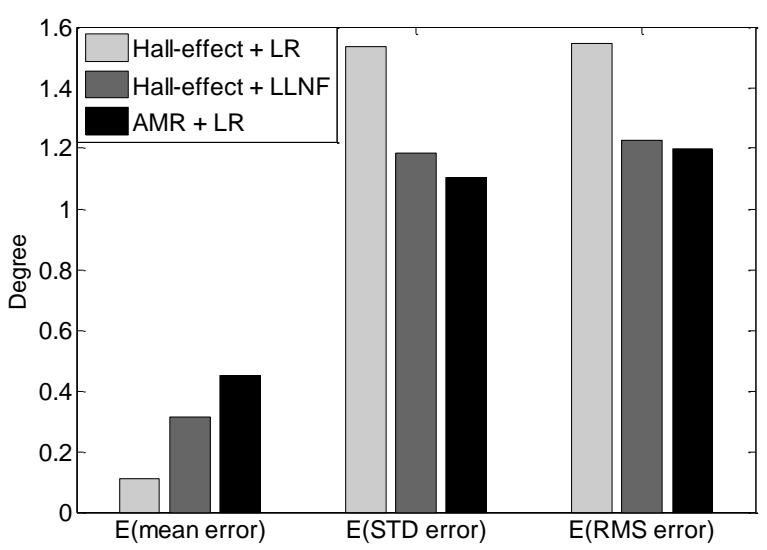

Figure 9. Comparison of three different estimators averaged performances over two different simulated gaits in the knee simulator.

over two different test gait patterns. The neuro-fuzzy estimator outperformed the linear regression estimator, on the train data with an RMS error of $0.46^{\circ}$ and on both test gait patterns with RMS errors of $1.29^{\circ}$ and $1.16^{\circ}$. These results demonstrate

TABLE I. COMPARISONS OF PERFORMANCES ON TRAIN AND TEST DATA FOR LINEAR REGRESSION ESTIMATES OF FE ANGLES BASED HALL-EFFECT SENSORS. THE TEST DATA CONSISTED OF OVERGROUND (GAIT1) AND TREADMILL WALKING (GAIT2) WHICH REPLICATED IN ROBOTIC KNEE SIMULATOR AT 30\% AND 100\% OF NATURAL SPEED (FREQUENCY).

\begin{tabular}{cc:ccccc}
\hline \hline & Train data & \multicolumn{4}{c}{ Test data } \\
\hline FE angles & $\begin{array}{c}\text { Squat } \\
\text { movement }\end{array}$ & $\begin{array}{c}\text { Gait1 } \\
30 \% \text { speed }\end{array}$ & $\begin{array}{c}\text { Gait1 } \\
\text { full speed }\end{array}$ & $\begin{array}{c}\text { Gait2 } \\
30 \% \text { speed }\end{array}$ & $\begin{array}{c}\text { Gait2 } \\
\text { full speed }\end{array}$ \\
\hline E(error) & $0.00^{\circ}$ & $0.24^{\circ}$ & $0.27^{\circ}$ & $-0.01^{\circ}$ & $-0.05^{\circ}$ \\
\hline STD(error) & $0.89^{\circ}$ & $1.26^{\circ}$ & $1.74^{\circ}$ & $0.83^{\circ}$ & $1.33^{\circ}$ \\
\hline RMS(error) & $0.89^{\circ}$ & $1.28^{\circ}$ & $1.76^{\circ}$ & $0.83^{\circ}$ & $1.33^{\circ}$ \\
\hline $\mathrm{R}^{2}$ & 0.99 & 0.99 & 0.99 & 0.99 & 0.99 \\
\hline \hline
\end{tabular}

TABLE II. COMPARISONS OF PERFORMANCES ON TRAIN AND TEST DATA FOR LOCALLY LINEAR NEURO-FUZZY ESTIMATES OF FE ANGLES BASED HALL-EFFECT SENSORS. THE TEST DATA CONSISTED OF OVERGROUND (GAIT1) AND TREADMILL WALKING (GAIT2) WHICH REPLICATED IN ROBOTIC KNEE SIMULATOR AT 30\% AND 100\% OF NATURAL SPEED (FREQUENCY).

\begin{tabular}{cc:cccc}
\hline \hline & Train data & \multicolumn{4}{c}{ Test data } \\
\hline FE angles & $\begin{array}{c}\text { Squat } \\
\text { movement }\end{array}$ & $\begin{array}{c}\text { Gait1 } \\
\text { 30\% speed }\end{array}$ & $\begin{array}{c}\text { Gait1 } \\
\text { full speed }\end{array}$ & $\begin{array}{c}\text { Gait2 } \\
30 \% \text { speed }\end{array}$ & $\begin{array}{c}\text { Gait2 } \\
\text { full speed }\end{array}$ \\
\hline E(error) & $0.00^{\circ}$ & $0.27^{\circ}$ & $0.32^{\circ}$ & $0.31^{\circ}$ & $0.31^{\circ}$ \\
\hline STD(error) & $0.46^{\circ}$ & $0.68^{\circ}$ & $1.25^{\circ}$ & $0.50^{\circ}$ & $1.12^{\circ}$ \\
\hline RMS(error) & $0.46^{\circ}$ & $0.73^{\circ}$ & $1.29^{\circ}$ & $0.59^{\circ}$ & $1.16^{\circ}$ \\
\hline $\mathrm{R}^{2}$ & $0.99^{\circ}$ & 0.99 & 0.99 & 0.99 & 0.99 \\
\hline \hline
\end{tabular}


the flexibility and generalization power of the neuro-fuzzy model to estimate FE angles from the sensors' raw data. The largest errors of Hall-effect based linear regression appeared in small knee angles below $25^{\circ}$ which can be seen as inaccurate estimation of minimum valleys of the gait pattern (Fig 7). However all extremums of gait patterns were accurately estimated by the LLNF. LLNF did not show noticeable inferior performance at extreme angles when comparing to intermediate ranges. In our previous study [16], linear regression based estimates from AMR sensors provided excellent performance on manually simulated movements with a total consumption of $69 \mathrm{~mW}$. The use of Hall-effect sensors can decrease the power consumption to only $20 \mathrm{~mW}$ in expense of a lower SNR and consequently lower accuracy when using similar estimators. In this study we investigated the possibility of improving FE estimate based on Hall-effect sensors using the neuro-fuzzy model. After testing both sensor configurations with their corresponding estimators in the robotic knee simulator, the comparison of the results proved the proximity of the performances. Linear regression based AMR sensors performed slightly better on both gait patterns, however the differences of STD error and RMS error calculated between both estimators are below $0.1^{\circ}$. Therefore, the neuro-fuzzy estimator built for Hall-effect sensors achieves an accurate and precise FE angle estimation, comparable to the performance of linear regression based AMR sensors with much lower power consumption.

The LLNF was chosen primarily due to the observed piecewise linear output of sensors to the measured flexion angle. Any other machine learning estimator such as multilayer neural networks or Gaussian linear regression that can extract such nonlinear mapping can perform well. LLNF was also providing a level of intuitiveness and transparency which allows detecting the subregions in measurement space that needs to be modeled with more complexity. The sensitivity of the magnetic sensors themselves can possibly be improved by designing specific magnetic concentrators [23] which could be integrated into the PE as well.

Hall-effect sensors display several advantages over AMR sensors. In addition to their general lower consumption, they have more compatibility with silicon technology and can therefore be realized on a single circuit board integrated in PE which leads to a substantial gain of space. The smaller size of Hall-effect sensors $\left(2.7 \times 2.35 \times 0.95 \mathrm{~mm}^{3}\right)$, used in this study, in comparison with the previously used AMR sensors $\left(4.8 \times 3.8 \times 1.4 \mathrm{~mm}^{3}\right)$ is a benefit for packaging as well. Very small Hall elements however suffer from higher offset and low frequency noise, and the choice of Hall-effect sensors need to be made with caution [24].

This study contributed also to the improvement of the validation procedure for the instrumented prostheses by introducing a robotic knee simulator. Indeed, by fixing the PE insert with integrated Hall-effect sensors into the robotic knee simulator, human-like walking gait patterns can be performed and tested. Previous studies used only numerical simulations [19] or manual knee simulators to validate estimators' performances [16], [25]. However none of those techniques could closely simulate realistic gait patterns, which means that the reported errors cannot reflect the performance for normal walking condition. In contrast, the presented validation with the robotic knee simulator can provide the realistic performance evaluation of the system. Due to the limitation of currently realized knee simulator, only the FE angle estimation was studied. Further studies have to be done to investigate kinematics estimations with a focus on 3D kinematics which requires an increase in controllable degrees of freedom of the simulator. Only two subjects' gait patterns were used in this study. Although the obtained performances are valid only for these two subjects, the ranges of flexion angle, i.e. $46^{\circ}$ and $57^{\circ}$, were equal or larger than the knee angle ranges obtained from a recent fluoroscopic study on 19 subjects walking on the treadmill (approximate FE range: $42 \pm 5^{\circ}$ ) [10]. More subjects and other activities could be still simulated in the robotic knee for validating the smart prosthesis for any population group or against other activities.

This work focused on a low-power kinematic measurement system, including the sensors and different estimators, and its validation in a robotic knee simulator, however other aspects of the design of the smart knee prosthesis, e.g. the remote powering, wireless communication, analogue front-end, force sensors, and packaging are in progress [18], [26]-[28]. Considering the smaller size, lower power consumption and the silicon technology compatibility of the proposed solution, it would promote the fabrication of a smart capsule, including also the necessary electronics, to be integrated into the polyethylene inserts of the commercially-available knee prostheses of different types for in-vivo post-operative monitoring of the patients.

\section{REFERENCES}

[1] G. Peat, R. McCarney, and P. Croft, "Knee pain and osteoarthritis in older adults: A review of community burden and current use of primary health care," Ann. Rheum. Dis., vol. 60, no. 2, pp. 91-97, 2001.

[2] L. Murphy, T. A. Schwartz, C. G. Helmick, J. B. Renner, G. Tudor, G. Koch, A. Dragomir, W. D. Kalsbeek, G. Luta, and J. M. Jordan, "Lifetime risk of symptomatic knee osteoarthritis," Arthritis Care Res., vol. 59, no. 9, pp. 1207-1213, 2008.

[3] A. J. Carr, O. Robertsson, S. Graves, A. J. Price, N. K. Arden, A. Judge, and D. J. Beard, "Knee replacement," The Lancet, vol. 379, no. 9823, pp. 1331-1340, Apr. 2012.

[4] D. D. D'Lima, S. Patil, N. Steklov, J. E. Slamin, and C. W. Colwell, "Tibial Forces Measured In Vivo After Total Knee Arthroplasty," $J$. Arthroplasty, vol. 21, no. 2, pp. 255-262, Feb. 2006.

[5] B. Heinlein, I. Kutzner, F. Graichen, A. Bender, A. Rohlmann, A. M. Halder, A. Beier, and G. Bergmann, "ESB clinical biomechanics award 2008: Complete data of total knee replacement loading for level walking and stair climbing measured in vivo with a follow-up of 6-10 months," Clin. Biomech., vol. 24, no. 4, pp. 315-326, May 2009.

[6] B. Kirking, J. Krevolin, C. Townsend, C. W. Colwell Jr., and D. D. D'Lima, "A multiaxial force-sensing implantable tibial prosthesis," $J$. Biomech., vol. 39, no. 9, pp. 1744-1751, 2006.

[7] I. Kutzner, B. Heinlein, F. Graichen, A. Bender, A. Rohlmann, A. Halder, A. Beier, and G. Bergmann, "Loading of the knee joint during activities of daily living measured in vivo in five subjects," $J$. Biomech., vol. 43, no. 11, pp. 2164-2173, Aug. 2010.

[8] A. Leardini, L. Chiari, U. D. Croce, and A. Cappozzo, "Human movement analysis using stereophotogrammetry: Part 3. Soft tissue 
artifact assessment and compensation," Gait Posture, vol. 21, no. 2, pp. 212-225, Feb. 2005.

[9] R. Stagni, S. Fantozzi, A. Cappello, and A. Leardini, "Quantification of soft tissue artefact in motion analysis by combining 3D fluoroscopy and stereophotogrammetry: a study on two subjects," Clin. Biomech., vol. 20, no. 3, pp. 320-329, Mar. 2005.

[10] A. Barré, J.-P. Thiran, B. M. Jolles, N. Theumann, and K. Aminian, "Soft tissue artifact assessment during treadmill walking in subjects with total knee arthroplasty," IEEE Trans. Biomed. Eng., vol. 60, no. 11, pp. 3131-3140, Jun. 2013.

[11] J. Favre, B. M. Jolles, R. Aissaoui, and K. Aminian, "Ambulatory measurement of 3D knee joint angle," J. Biomech., vol. 41, no. 5, pp. 1029-1035, 2008.

[12] H. Dejnabadi, B. M. Jolles, and K. Aminian, "A new approach to accurate measurement of uniaxial joint angles based on a combination of accelerometers and gyroscopes," IEEE Trans. Biomed. Eng., vol. 52, no. 8, pp. 1478-1484, 2005.

[13] L. Kun, Y. Inoue, K. Shibata, and C. Enguo, “Ambulatory Estimation of Knee-Joint Kinematics in Anatomical Coordinate System Using Accelerometers and Magnetometers," IEEE Trans. Biomed. Eng., vol. 58, no. 2, pp. 435-442, 2011.

[14] S. Bonnet and R. Heliot, "A Magnetometer-Based Approach for Studying Human Movements," IEEE Trans. Biomed. Eng., vol. 54, no. 7, pp. 1353-1355, 2007.

[15] D. Roetenberg, P. J. Slycke, and P. H. Veltink, "Ambulatory Position and Orientation Tracking Fusing Magnetic and Inertial Sensing," IEEE Trans. Biomed. Eng., vol. 54, no. 5, pp. 883-890, 2007.

[16] A. Arami, A. Vallet, and K. Aminian, "Accurate Measurement of Concurrent Flexion-Extension and Internal-External Rotations in Smart Knee Prostheses," IEEE Trans. Biomed. Eng., vol. 60, no. 9, pp. 2504-2510, 2013.

[17] A. Arami, M. Simoncini, O. Atasoy, S. Ali, W. Hasenkamp, A. Bertsch, E. Meurville, S. Tanner, P. Renaud, C. Dehollain, P. Farine, B. M. Jolles, K. Aminian, and P. Ryser, "Instrumented Knee Prosthesis for Force and Kinematics Measurements," IEEE Trans. Autom. Sci. Eng., vol. 10, no. 3, pp. 615-624, 2013.

[18] O. Atasoy and C. Dehollaini, "Remote powering realization for smart orthopedic implants," in New Circuits and Systems Conference (NEWCAS), 2012 IEEE 10th International, 2012, pp. 521-524.

[19] A. Arami, A. Barre, R. Berthelin, and K. Aminian, "Estimation of prosthetic knee angles via data fusion of implantable and wearable sensors," in 2013 IEEE International Conference on Body Sensor Networks (BSN), 2013, pp. 1-6.

[20] B. J. Fregly, T. F. Besier, D. G. Lloyd, S. L. Delp, S. A. Banks, M. G. Pandy, and D. D. D'Lima, "Grand challenge competition to predict in vivo knee loads," J. Orthop. Res., vol. 30, no. 4, pp. 503-513, 2012

[21] O. Nelles, "Nonlinear System Identification," Meas. Sci. Technol., vol. 13, no. 4, p. 646, Apr. 2002.

[22] O. Nelles and R. Isermann, "Basis function networks for interpolation of local linear models," in, Proceedings of the 35th IEEE Conference on Decision and Control, 1996, 1996, vol. 1, pp. 470-475 vol.1.

[23] P. Leroy, C. Coillot, A. F. Roux, and G. M. Chanteur, "High magnetic field amplification for improving the sensitivity of Hall sensors," IEEE Sens. J., vol. 6, no. 3, pp. 707-713, Jun. 2006.

[24] C. Schott, R. Racz, and S. Huber, "Smart CMOS sensors with integrated magnetic concentrators," in 2005 IEEE Sensors, 2005, p. 4 pp.-.

[25] A. Arami, J. D. Rechenmann, and K. Aminian, "Reference-Free Automated Magnetic Sensor Calibration for Angle Estimation in Smart Knee Prostheses," IEEE Sens. J., vol. 14, no. 6, pp. 1788-1796, Jun. 2014.

[26] O. Atasoy and C. Dehollain, "Full-duplex communication and remote powering implementation of an electronic knee implant," in Research in Microelectronics and Electronics (PRIME), 2013 9th Conference on Ph.D, 2013, pp. 217-220.

[27] S. Tanner, S. Ali, M. Banjevic, A. Arami, K. Aminian, W. Hasenkamp, A. Bertsch, P. Renaud, and P.-A. Farine, “An Analog Front-End and ADC Integrated Circuit for Implantable Force and Orientation Measurements in Joint Prosthesis," in Wireless Mobile Communication and Healthcare, B. Godara and K. S. Nikita, Eds. Springer Berlin Heidelberg, 2013, pp. 295-302.

[28] W. Hasenkamp, N. Thevenaz, J. Villard, A. Bertsch, A. Arami, K. Aminian, A. Terrier, and P. Renaud, "Design and test of a MEMS strain-sensing device for monitoring artificial knee implants," Biomed. Microdevices, vol. 15, no. 5, pp. 831-839, Oct. 2013.

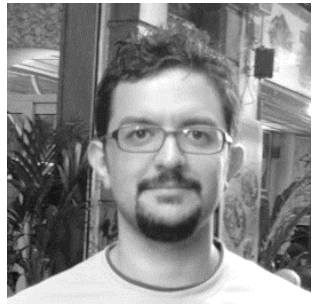

Arash Arami received his B.Sc., M.Sc. and Ph.D. degrees from University of Tabriz, University of Tehran, and Ecole Polytechnique Fédérale de Lausanne (EPFL) in 2006, 2009, and 2014 respectively all in electrical engineering. He was a research assistant and then a research associate at Laboratory of Movement Analysis and Measurement of EPFL from 2009 to 2015 . He is currently a research associate at human robotics group in Imperial College London, UK. His main research interests are intelligent assistive systems, motor neuroprosthetics, biomedical signal processing and system identification.

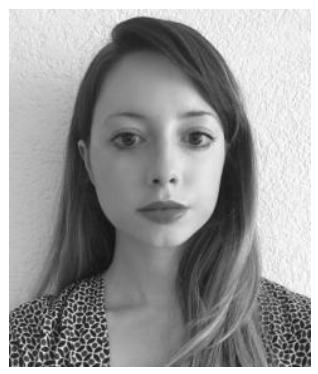

Natacha Vida Martins was born in Neuchâtel, Switzerland in 1990. She received the master's degree in biomechanics from Ecole Polytechnique Fédérale de Lausanne, Switzerland, in 2015. Her main research interests are clinical orthopaedics and orthopeadic implants.

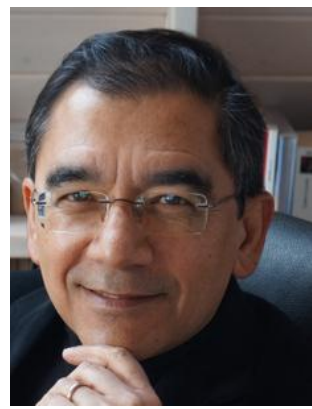

Kamiar Aminian received the M.S. degree in electrical engineering and the Ph.D degree in biomedical engineering in 1989 from Ecole Polytechnique Fédérale de Lausanne (EPFL), Lausanne, Switzerland. He is currently Professor in the Institute of Bioengineering and the director of the Laboratory of Movement Analysis and Measurement of EPFL. His research focuses on methodologies for human movement monitoring and analysis in real world conditions mainly based on wearable technologies, with emphasis on gait, physical activity and sport. His research aims to perform outcome evaluation in orthopaedics, to improve motor function and intervention programs in aging and patients with movement disorders and pain, and to identify metrics of performance in sport science. He is teaching in the areas of physiology and instrumentation, medical devices, biomechanics, and sports. $\mathrm{He}$ is author or co-author of more than 450 scientific papers published in reviewed journals and presented at international conferences and holds 8 patents related to medical devices. 